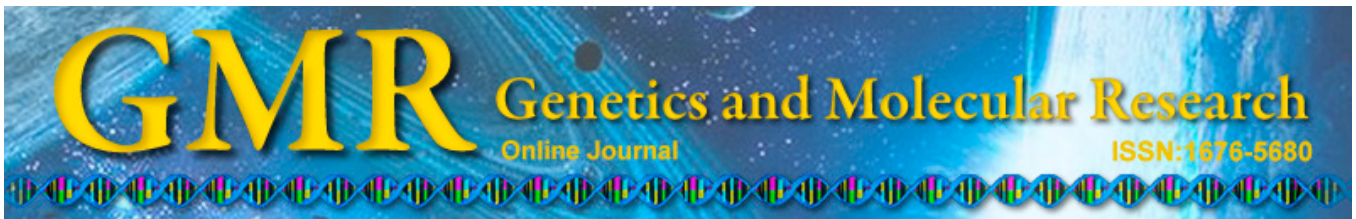

\title{
Effects of Herceptin on circulating tumor cells in HER2 positive early breast cancer
}

\author{
J.-L. Zhang, Q. Yao, J.-H. Chen ,Y. Wang, H. Wang, Q. Fan, R. Ling, \\ J. Yi and L. Wang
}

Xijing Hospital Vascular Endocrine Surgery, Xi'an, China

Corresponding author: J.-L. Zhang

E-mail: zhangjuliang_1@yeah.net

Genet. Mol. Res. 14 (1): 2099-2103 (2015)

Received April 15, 2014

Accepted October 20, 2014

Published March 20, 2015

DOI http://dx.doi.org/10.4238/2015.March.20.20

\begin{abstract}
The objective of this study was to determine the changes in peripheral blood circulating tumor cells in HER2-positive early breast cancer before and after Herceptin therapy, and to explore the effects of the HER2 gene and Herceptin on circulating tumor cells. CK19 mRNA expression in peripheral blood was evaluated by qRTPCR as an index of circulating tumor cells in 15 cases of HER-2positive breast cancer and 18 cases of HER2-negative breast cancer before, and after chemotherapy as well. Ten cases of HER2-positive breast cancer continued on Herceptin therapy for 3 months after chemotherapy, and their peripheral blood was again drawn and assayed for CK-19 mRNA expression. Preoperatively, all cases of HER2positive cancer were positive for CK19 mRNA in peripheral blood, but 6 cases of HER2-negative breast cancer were positive (33.3\%), where there was a substantial difference between the two groups. After 6 cycles of adjuvant chemotherapy, CK19 positive rates in cases of HER2-positive and -negative breast cancer reduced by 93.3 and $11.1 \%$, respectively, with a significant difference still existing. After 3 months of Herceptin therapy, expression of CK19 mRNA declined
\end{abstract}


considerably in 10 cases of HER2 positive breast cancer (113.66 \pm 88.65 vs $63.35 \pm 49.27, \mathrm{P}=0.025)$. HER-2 gene expression closely correlated with circulating tumor cells in peripheral blood of early breast cancer patients. Moreover, Herceptin, a monoclonal antibody for HER2, can reduce the number of circulating tumor cells, which can be an early predictive factor for Herceptin therapy effectiveness against breast cancer.

Key words: Herceptin; Breast cancer; Circulating tumor cells

\section{INTRODUCTION}

The expression of HER2 genes is the most important prognostic indicator of early breast cancer. Studies indicate that HER2-positive cases have lower disease-free overall survival compared to HER2-negative patients. The existence of circulating tumor cells (CTCs) in peripheral blood of early breast cancer patients is a vital factor for occurrence of metastases, of which CTCs is an independent prognostic index (Dawood et al., 2008). Although the meaning of CTCs is still unclear in the operation of breast cancer, an investigation has demonstrated that the variation in CTCs could be an early indicator of adjuvant chemotherapy effectiveness (Zhang et al., 2008). In cases of early breast cancer, we still need further experimental data to prove whether CTCs correlate with the expression of the HER2 gene. Thus, we used the epithelial cell-specific gene cytokeratin 19 (CK19) as a marker to investigate the relationship between HER-2-positive early breast cancer and CTCs, and we discuss the effect of Herceptin on CTCs, providing guidance in evaluating the therapeutic effect of Herceptin.

\section{MATERIAL AND METHODS}

\section{Clinical materials and sample collections}

Prior to surgical resection, peripheral blood samples were obtained from early breast cancer patients in our hospital from 2007 to 2010; preoperative biopsy was done and tissue pathology verified, while immunohistochemical $\left(3^{+}\right)$or FISH confirmed HER2gene positive expression. On the basis of HER-2 expression, the patients were divided into HER2-positive and -negative groups, and the common conditions and pathologic features of the cases in the two groups are shown in Table 1. Statistical analysis showed no substantial differences between the groups. Venous blood samples were obtained from the two groups of patients before surgery and 1 month after chemotherapy $\left(75 \mathrm{mg} / \mathrm{m}^{2}\right.$ docetaxel, day $1 ; 90 \mathrm{mg} / \mathrm{m}^{2}$ epirubicin, day 1 , every 21 days, total of 6 cycles), and stored at $-80^{\circ} \mathrm{C}$ until RNA extraction. Ten cases of HER2-positive breast cancer remained on Herceptin therapy $(110 \mathrm{mg} /$ week) for 1 month after chemotherapy, with the same method being used to collect the samples. Patients provided signed informed consent before specimens were collected. 


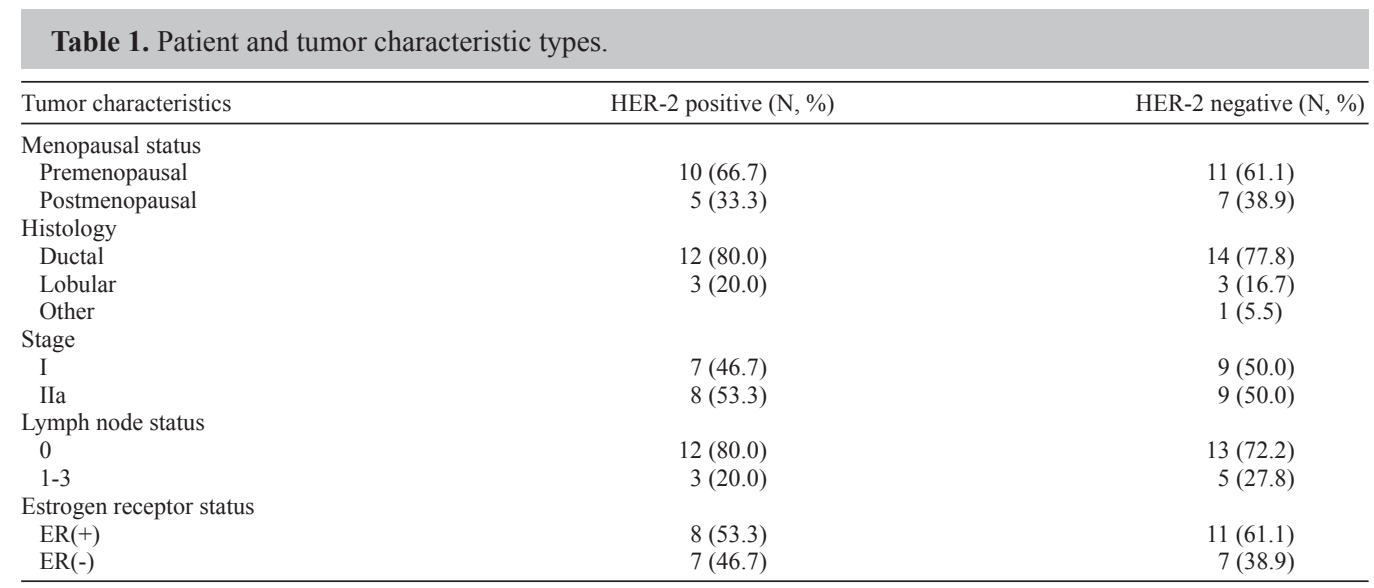

\section{Real time RT-PCR}

Trizol RNA extraction kits were purchased from Promogac Company (CA, USA). According to the manual of reverse transcription kits (Biotek, Beijing, China), total RNA was extracted, and mRNA was reverse transcribed into cDNA. Primers were designed and synthesized: CK19- forward: 5'-ATGCGAAGCCAATATGAGGT-3' and reverse: 5'-AGTAACCTCGGACCTGCTCA-3'; $\beta$-actin primer- forward: 5'CCTTCCTGGGTATGGAATCCT-3' and reverse: 5'-GGAGCAATGATCTTGATCTT-3'. The primers were produced by Shanghai Shenggong Company (Shanghai, China). PCR was carried out in $25 \mu \mathrm{L}$, including $0.5 \mu \mathrm{L}$ reverse transcription mix, $12.5 \mu \mathrm{L}$ SYBR Green PCR Mastermix (Bioteck, Beijing, China), $0.5 \mu \mathrm{L} 10 \mathrm{mM}$ forward and reverse primer, and DEPC water to $25 \mu \mathrm{L}$. The reaction conditions were as follows: pre-denaturation at $95^{\circ} \mathrm{C}$ for $10 \mathrm{~s}$ and 40 cycles of denaturation at $95^{\circ} \mathrm{C}$ for $5 \mathrm{~s}$, and annealing at $60^{\circ} \mathrm{C}$ for $34 \mathrm{~s}$. Quantitative real-timer PCR was performed in an ABI 7500 system, where the relative concentration of CK19 cDNA was determined by the threshold cycle method for each sample, according to manufacturer instructions.

\section{Statistical analysis}

The SPSS10.0 statistical software was used, where comparison between the two groups was done with the rank sum test or $\chi^{2}$ test, $\mathrm{P}<0.05$ indicated a significant difference.

\section{RESULTS}

\section{CK19 expression in peripheral blood before surgery and after chemotherapy}

Before the operation, all cases of HER2-positive cancers were positive for CK19 mRNA in peripheral blood, but 6 cases of HER 2 negative breast cancer were positive (33.3\%), which was a substantial difference between the two groups $(P=0.000)$ (Table 2). After 6 cycles of adjuvant chemotherapy, 14 cases of HER2 positive breast cancer rendered positive 
results $(93.3 \%)$, while only 2 negative cases expressed positive results, with the difference still being significant. Peripheral blood expression was similar in breast cancer with distinctive pathological features and different lymphatic metastasis and hormone receptor status.

Table2. Expression of peripheral blood CK19 pre-operation and post-chemotherapy.

\begin{tabular}{lcc}
\hline & \multicolumn{2}{c}{ Positive expression of CK19 (N, \%) } \\
\cline { 2 - 3 } & Pre-operation & Post-chemotherapy \\
\hline HER-2 positive & $15(100)^{*}$ & $14(93.3)^{*}$ \\
HER-2 negative & $6(33.3)$ & $2(11.1)$ \\
\hline
\end{tabular}

*Compared with HER-2 negative cases, the difference has statistical meaning. Significant difference was found between HER-2 positive and negative groups.

\section{Influence of Herceptin on the expression of CK19 in peripheral blood of HER2- positive breast cancer patients}

Ten cases of HER-2-positive breast cancer accepted Herceptin therapy. After 3 months of Herceptin therapy, the expression of CK19 mRNA declined considerably in the 10 cases of HER-2-positive breast cancer (113.66 \pm 88.65 vs $63.35 \pm 49.27, \mathrm{P}=0.025)$. On the other hand, in 5 cases without Herceptin therapy compared with 1 month of chemotherapy, the expression of CK19 did not vary notably $(78.20 \pm 69.85$ vs $70.35 \pm 59.77, \mathrm{P}=0.155)$ (Table 3 ).

Table 3. Variation of CK19 expressions in HER-2 positive patients accepted Herceptin therapy.

\begin{tabular}{llc}
\hline Type $(\mathrm{N})$ & \multicolumn{2}{c}{ Expression index of peripheral blood CK19 (means \pm SD) } \\
\cline { 2 - 3 } & Pre-treatment & 3 months post-treatment \\
\hline Herceptin group (10) & $81.66 \pm 68.65^{*}$ & $23.35 \pm 19.27$ \\
Without Herceptin group (5) & $78.20 \pm 69.85$ & $70.35 \pm 59.77$ \\
\hline *Compared 3-month therapy, the difference has statistical meaning. In herception group, significant difference was \\
found between pre-treatment and post-treatment patients.
\end{tabular}

\section{DISCUSSION}

Among the prognostic factors for breast cancer, including tumor size, lymph node status, hormone receptor status, HER2 gene expression, etc., especially for early stage breast cancer, HER2 gene expression is the most important independent prognostic factor. Numerous studies confirm that in various malignant tumors, including breast cancer, hematogenous spread can occur early. Cytokeratin 19 (CK19) is a protein strictly specific for epithelial cells, showing negative expression in non-epithelial tissue or cells such as normal blood, bone marrow and lymph nodes. Therefore, CK19 can be used as the micrometastasis marker for checking if circulating tumor cells are present in the blood of cancer patients (Datta et al., 1994). Giuliano et al. (2011) demonstrated in metastatic breast cancer that the number of circulating tumor cells in peripheral blood cannot only be an indicator of prognosis, but can also be used to predict the efficacy of first-line chemotherapy to provide individualized treatment options for patients. Although among operable breast cancers, there is a lack of reliable evidence verifying CTCs as a prognostic indicator, research has shown that in ovarian cancer and colon cancer, high levels of preoperative CTCs in peripheral blood was significantly lower over- 
all survival. Meanwhile, after surgery and chemotherapy reducing CTCs indicates a better prognosis (Negin et al., 2011; Aktas et al., 2011a). Lang et al. (2009) and Aktas et al. (2011b) demonstrated that the number of CTCs in HER-2-positive operable breast cancer cases was significantly higher than in negative cases. This indicated that HER2 gene expression may enhance the spread of cancer cells into the blood, regardless of primary tumor hormone receptor status, while circulating tumor cells in peripheral blood were found to be mostly hormone receptor-negative. Our results also showed that, in early breast cancer, the positive expression of CTCs in HER2-positive patients was considerably higher than in the HER2-negative patients. Especially after the end of adjuvant chemotherapy, HER2-positive CTCs in peripheral blood of patients still remained at high expression rates, while HER-2-negative CTCs in patients was markedly reduced, implying the expression of HER-2 gene is closely related to CTCs.

In the treatment of breast cancer, neoadjuvant chemotherapy, adjuvant chemotherapy and targeted therapy have a lack of effective biological indicators. Our previous studies confirmed that the number of CTCs in peripheral blood can be used as an index of the early efficacy of novel neoadjuvant chemotherapy (Lang et al. 2009). In this study, 10 HER2-positive patients were treated with Herceptin for 3 months, which caused a marked decrease in the number of CTCs. By contrast, the expression of CK19 in the peripheral blood did not significantly change for 5 cases who did not receive Herceptin therapy. This indicated that CTCs in Herceptin therapy played an important role in determining drug effectiveness during the treatment. Picking CTCs in peripheral blood as an indicator, also has advantages such as sample can readily drawn, CTCs can be easily detected, and test be repeated simply. Obviously, more detailed clinical trials are still needed to see whether CTCs have a true prognostic value in HER-2-positive breast cancer, and if the course of Herceptin treatment can be based on changes in CTCs, and if the efficacy of Herceptin therapy can be accurately determined by monitoring CTCs.

\section{REFERENCES}

Aktas B, Kasimir-Bauer S, Heubner M, Kimmig R, et al. (2011a) Molecular profiling and prognostic relevance of circulating tumor cells in the blood of ovarian cancer patients at primary diagnosis and after platinum-based chemotherapy. Int. J. Gynecol. Cancer 21:822-830.

Aktas B, Muller V, Tewes M, Zeitz J, et al. (2011b) Comparison of estrogen and progesterone receptor status of circulating tumor cells and the primary tumor in metastatic breast cancer patients. Gynecol. Oncol. 122:356-360.

Datta YH, Adams PT, Drobyski WREthier SP, Terry VH, et al. (1994) Sensitive detection of occult breast cancer by the reverse-transcriptase polymerase chain reaction. J. Clin. Oncol. 12:475-482.

Dawood S, Broglio K, Valero V, Reuben J, et al. (2008) Circulating tumor cells in metastatic breast cancer: from prognostic stratification to modification of the staging. Cancer113:2422-2430.

Giuliano M, Giordano A, Jackson S Hess KR, De Giorgi U, et al. (2011) Circulating tumor cells as prognostic and predictive markers in metastatic breast cancer patients receiving first-line systemic treatment. Breast Cancer Res. 13:R67.

Lang JE, Mosalpuria K, Cristofanilli M, Krishnamurthy S, et al. (2009) HER2 status predicts the presence of circulating tumor cells in patients with operable breast cancer. Breast Cancer Res. Treat. 113:501-507.

Negin BP and Cohen SJ (2011) Circulating tumor cells in colorectal cancer: past, present, and future challenges. Curr. Treat. Options Oncol. 11:1-13.

Zhang JL, Wang L, Ling R, Yao Q, et al. (2008) Neoadjuvant chemotherapy in breast cancer micrometastases in peripheral blood before and after the change in cancer cells and its clinical significance. Clin. Oncol. China 15:45-48. 